\title{
Splenic melanosis in agouti and black mice*
}

\author{
Dominika Michalczyk-Wetula, Justyna Wieczorek and Przemysław M. Płonka ${ }^{\bowtie}$ \\ Department of Biophysics, Faculty of Biochemistry, Biophysics and Biotechnology, Jagiellonian University, Kraków, Poland
}

\begin{abstract}
An interesting example of extradermal deposition of melanin in vertebrates, notably in mammals, is splenic melanosis. In particular, if the phenomenon of splenic melanosis is correlated with hair or skin pigmentation, it must reflect the amount and perhaps the quality of pigment produced in hair follicle melanocytes. The present paper is our first study on splenic pigmentation in mice of phenotype agouti. We used untreated mixed background mice C57BL/6;129/SvJ (black - a/a, agouti $-A / a, A / A)$, and as a control - black C57BL/6 and agouti fur from 129/SvJ mice, Mongolian gerbils (Meriones unguiculatus) and golden hamsters (Mesocricetus auratus). After euthanasia skin and spleen was evaluated macroscopically, photographed and collected for further analysis using Fontana-Masson and hematoxylin-eosin staining and electron paramagnetic resonance (EPR) at $\mathrm{X}$-band. Spleens of the agouti mice revealed splenic melanosis but were slightly weaker pigmented than their black counterparts, while the presence of pheomelanin was difficult to determine. The fur of both phenotypes was of similar melanin content, with the same tendency as in the spleens. The contribution of pheomelanin in the agouti fur was on the border of detectability by EPR. Histological and EPR analysis confirmed the presence of melanin in the melanotic spleens. The shape of the EPR signal showed a dominance of eumelanin in fur and in melanized spleens in both phenotypes of mice. Therefore, splenic melanosis does reflect the hair follicle pigmentation not only in black, but also in agouti mice.
\end{abstract}

Key words: agouti, EPR, hair cycle, melanin, spleen

Received: 13 April, 2015; revised: 05 May, 2015; accepted: 21 May, 2015; available on-line: 20 August, 2015

\section{INTRODUCTION}

From the point of view of contemporary biochemistry melanin is a very interesting biopolymer - it is produced by polymerization of amino acids or their derivatives, it reveals a very complicated and so far not well understood micro- and mesoscopic structure (Meredith \& Sarna, 2006), yet there is no information about the primary structure (sequence of amino acids) of melanin coded in the genomes of melanin-producing organisms. Obviously, and unlike proteins, there is no RNA for melanin in the transcryptome, as melanin is synthesized beyond the translation-related pathway of synthesis of proteins. Consequently, while still being a close relative of proteins, it reserves a very special place in the metabolome, close to, but apart from the proteome.

Despite this unique position, there are many melanogenesis-related genes, which encode mainly the responsible enzymes, their transcription factors (e.g. microphthal- mia transcription factor - MiTF; Vachtenheim \& Borovansky, 2010), and proteins which regulate the structure and functioning of melanosomes (Slominski et al., 2004, 2005b, Schallreuter et al., 2008). Therefore, the melanin functions are indirectly encoded in the genome. Among them the most important is their function as a pigment responsible for coloration of the body (skin and its appendages), camouflage and signaling (Wood et al., 1999; Plonka et al., 2009). And despite the majority of the melanogenesis-related reactions running non-enzymatically (Land et al., 2004), the body coloration still depends on many genes (in mice their number is estimated at more than 150 alleles encrypted in over 90 loci; Slominski et al., 2005b).

Agouti protein is an antagonist and a reverse-agonist of the melanocyte-stimulating hormone (MSH) receptor type 1 (MC1R) (Ollmann et al., 1998). Its binding to the receptor strongly inhibits the process of melanin synthesis (Slominski et al., 2004, 2005b), and most probably switches it on pheomelanogenesis (Oyehaug et al., 2002). As a consequence, if acting periodically in a hair follicle (secreted by the dermal papilla fibroblasts, Milliar et al., 1995), it causes a typical "band" coloration of the hair shaft and a cryptic coloration of the whole hair coat called "agouti" (Sakurai et al., 1975). The "agouti" phenotype is commonly called the "wild" phenotype of the coloration (Oyehaug et al 2002, Slominski et al., 2004, 2005b).

Splenic melanosis discovered in black C57BL/6 mice (Weissman, 1967), and so far attributed almost exclusively to the black mice (Linden et al., 2012) is an intriguing phenomenon related to the mammalian melanogenesis (van der Heijden et al., 1995). It is probably a manifestation of extradermal melanogenesis observed in visceral organs of various vertebrates (Zieri et al., 2015). We have suggested that in mice it may be somehow related to the skin melanogenesis and hair cycling (Plonka et al., 2005; Michalczyk et al., 2009; Michalczyk-Wetula et al., 2013). Perhaps a part of melanin synthesized in the follicular melanocytes is transferred to the spleen, where

e-mail: przemyslaw.plonka@uj.edu.pl

*Preliminary report of this work was presented by J.W. at 42nd Winter School of the Faculty of Biochemistry, Biophysics and Biotechnology of the Jagiellonian University "From Genome to Proteome", February 10-14, 2015, Zakopane, Poland

Abbreviations: 129/SvJ, inbred mouse strain developed from 129 strains by LC Stevens, originating from the Jackson Laboratory's Colony; AHP, aminohydroxyphenylalanine; C57BL/6, Cross 57 th-generation female $\times 52$ nd-genertion male black, 6 th substrain (inbred mouse strain); dopa, 3,4-dihydroxyphenylalanine; DPPH, 1,1-diphenyl-2-picrylhydrazyl; EPR, electron paramagnetic resonance; ESR, electron spin resonance; FM, Fontana-Masson; $\mathrm{HE}$, hematoxylin and eosin; MC1R, melanocyte-stimulating hormone receptor type 1 ; MiTF, microphthalmia transcription factor; MSH, melanocyte-stimulating hormone; PTCA, pyrrole-2,3,5-tricarboxylic acid 
it may undergo a slow degradation. Interestingly, black mice reveal genotype $a / a$ in locus agouti, and do not produce agouti protein (Bultman et al., 1991; Slominski et al., 2005a).

We have shown that in C57BL/6 mice the degree of melanosis depends on the age of mice (Michalczyk et al., 2009), and that in the young mice (below 6 months of life) it is synchronized with the hair cycle (Plonka et al., 2005; Michalczyk-Wetula et al., 2013). The hair cycle is a very interesting physiological phenomenon of a periodical remodeling of tissues producing the hair shaft along with its pigments, which in young mice reveals a wavelike, locally synchronized character (Dry 1926; Chase \& Eaton, 1959). It is regulated with a strong support from the immunological system (Paus et al., 1999), thus it is not surprising that the actual state of the spleen and the actual stage of the hair cycle are interdependent (Slominski et al., 1997). Further study on the relation between spleen, hair cycle and the immune system may reveal new facts on the systemic effects of hair growth. Among them, the splenic melanosis remains an important target for the research.

So far, almost all the studies concerned black mice. While only in one case the authors checked other phenotypes, which were reported almost exclusively not to manifest splenic melanosis (Crichton et al., 1978), there exists a general claim that the phenomenon is typical of black mice (Linden et al., 2012). The intricate activity of agouti protein and the related regulators towards various types of MSH receptors in the central nervous system are manifested by systemic effects, such as obesity or diabetes (Kaelin et al., 2008). Therefore, to determine other systemic effects of the agouti protein it is important to learn whether the phenomenon of splenic melanosis depends on the genotype in locus agouti. If it is the case, it will confirm the hair pigmentation-dependent character of splenic melanosis. In the present paper we have analyzed splenic melanosis in the mice revealing the wild phenotype, therefore of $A / a$ or $A / A$ genotype in locus agouti, and we compare the amount and quality of splenic melanin to the black mice of $a / a$ genotype.

\section{MATERIAL AND METHODS}

Animals. C57BL/6;129/SvJ mixed background mice (Linder, 2003) with two phenotypes of fur color black (locus agouti genotype $a / a$ ) and agouti (locus agouti genotype $A / a$ and $A / A$ ) (Threadgill et al., 1997), in the age of 1-107 weeks, males and females were obtained from the local animal breeding facility of the Faculty of Biochemistry, Biophysics, and Biotechnology of the Jagiellonian University. All the mice were untreated, came from the breeding herd, and were not used for further reproduction. Mice were housed under conventional conditions in community cages and $12 \mathrm{~h}$ day/night regime with continuous access to food - standard laboratory rodent chow (Labofeed B provided by Morawski, Kcynia, Poland) and drinking water. The animals were handled with full respect to the ethical and legal standards and rules being in force during the observations.

The mice were weighed, euthanized (cervical dislocation in a deep anesthesia, xylazine - Sedazin ${ }^{\circledR}$, Biowet Puławy Sp. z o.o. Puławy, Poland, and ketamine - Ve$\operatorname{taKetam}^{\circledR}$, VetAgro, Lublin, Poland) whereupon the biological material was evaluated, photographed and collected. Biological material was harvested over almost four years (2007-2010).
Agouti fur of Mongolian gerbils Meriones unguiculatus (Milne-Edwards, 1867) and golden hamsters Mesocricetus auratus (Waterhouse, 1839), and of the 129/SvJ agouti mice came from the local collection of furs of the Department of Biophysics, Kraków, Poland (see Plonka et al., 2003; Slominski et al., 2005a; 2005b; Plonka 2009).

Analysis of spleen. The harvested spleens were photographed with a Nikon digital camera D80 equipped with Nikkor 18-70mm 1:3,5-4,5G ED AF-S DX lenses (Nikon DX SWM ED IF Aspherical ф67) (Nikon Corporation, Tokyo, Japan), weighed and carefully examined for macroscopic evidence of melanosis. If melanin was present, the area of melanosis was estimated as a percentage of the total spleen area. In the end of the observations the percentage of melanotic spleens per a given experimental group of mice was calculated. Selected spleens were fixed in buffered $5 \%$ formalin (phosphate buffer, $\mathrm{pH}=7.4$ ) and embedded in paraffin for histological analysis. The fixed spleens were cut into $5 \mu \mathrm{m}$ slices and stained with hematoxylin and eosin (HE) and according to Fontana-Masson (FM) procedure for melanin. Images of stained tissues were taken by a reverse Eclipse Ti microscope (Nikon) equipped with the Nis elements F 3.0 imaging software (Nikon). For details see Michalczyk-Wetula et al., 2013. Another part of spleens selected randomly from the group with melanosis was analysed by EPR spectroscopy.

Analysis of skin and fur. After euthanasia the back skin of each animal was separated on the level of subcutis, cut out, spread on a piece of transparent foil and photographed from the subcutis side using the Nikon digital camera D80 (see above). The important point is to keep the fur coat dry during photographing, because wet fur adheres to the upper skin surface and strongly affects its coloration seen from below. We found it to be a convenient way of a rough distinguishing between the whole back skin in telogen, in anagen and the skin in parts in various stages of the hair cycle, on the base of the back skin color from below (see Fig. 1). Next the skin was fixed in buffered 5\% formalin (phosphate buffer, $\mathrm{pH}=7.4)$. A part of black and agouti animals were shaved and the fur was collected for electron paramagnetic (spin) resonance (EPR, ESR) analysis.

Model substances. A powder sample of dopa melanin and cysteinyldopa melanin served as a standard to compare the EPR spectra of eu- and pheomelanin, respectively. For details see Wolnicka-Glubisz et al., 2012.

EPR analysis. EPR spectra of melanins in spleens and fur were recorded by an E-3 spectrometer (Varian, Sunnyvale, LA, USA) in a Wilmad finger quartz Dewar WG-816-B (Rototec-Spintec GmbH, Griesheim, Germa-

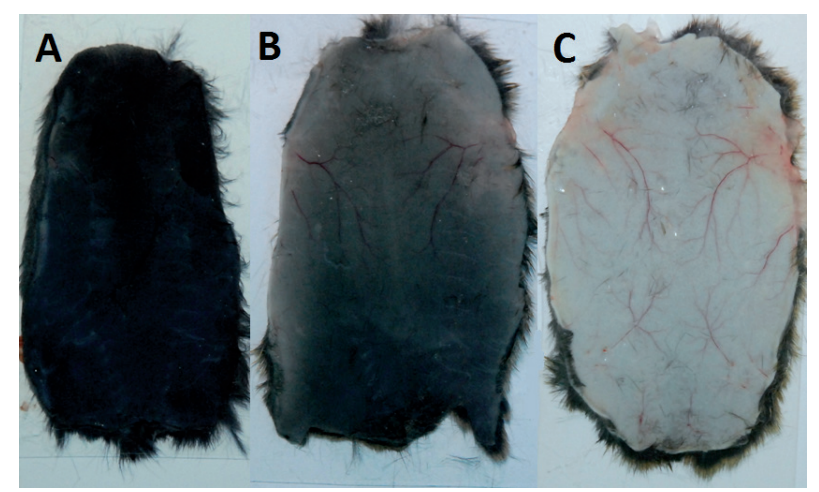

Figure 1. Mice back skin photographed from below.

$100 \%$ anagen $6(\mathbf{A})$, during anagen-catagen-telogen transition (B), $100 \%$ telogen $(\mathbf{C})$. 
ny), in liquid nitrogen $(77 \mathrm{~K})$ or at ambient temperature (fur, quantitative comparison) at X-band (ca. 9.2 GHz), $1 \mathrm{~mW}$ microwave power, 5 (synthetic melanin) or $10 \mathrm{Gs}$ modulation amplitude, receiver gain $5 \times 10^{3}-2 \times 10^{6}, 300 \mathrm{~s}$ scan time and $0.3 \mathrm{~s}$ time constant. For qualitative analysis, to avoid signal distorsion, we used modulation amplitude $0.5-1$ Gs and microwave power $0.5 \mathrm{~mW}$. Each spectrum of spleen was averaged 10 times, otherwise the gain and averaging were adjusted to particular needs. The fur samples weighed 20 (quantitative measurements) or 30 (qualitative measurements) $\pm 1 \mathrm{mg}$, and the powder melanin samples $-8.2 \pm 0.1 \mathrm{mg}$. For quantitative comparisons the amplitudes or integral intensities of signals were standardized per the same receiver gain. The signals of spleens were not standardized per constant mass, so their intensities (expressed as the EPR signal amplitudes) directly represent the absolute amount of melanin in the spleen.

Statistical evaluation of the data. Altogether we evaluated 884 mice (193 with black and 691 with agouti fur). As the breeding herd was primarily used for other purposes, the number of mice per a given time point (weeks after birth) was difficult to keep constant and to predict in the described study (1-20 black, and 1-63 agouti mice per a time period, on average about 6 black and 24 agouti mice per a time point). For the same reason, as well as because of the "yes-or-no" character of splenic melanosis, we were unable to systematically compare the melanization at every week post birth using statistic tools. In this case we were, however, able to show the linear trend lines for changing the percentage of melanotic spleens, and for changing the average area of melanosis in the melanotic spleens, with time. To exhibit the pooled results of the average area of melanosis we used the whole population of melanotic spleens $(n=38$ for black and 83 for agouti mice). For the quantitative EPR comparisons we used $n=15$ spleens for black and $\mathrm{n}=15$ for agouti mice (randomly selected from the group of the strongest pigmentation), $n=4$ samples of $129 / \mathrm{SvJ}$ agouti and $n=8$ of $C 57 \mathrm{BL} / 6$ black mice fur. We tested the statistical significance of the differences between variances with the $\mathrm{F}$ Snedecor test whereupon - the significance of the differences between the mean values with the independent, two-tail Student's $t$-test for groups of equal or non-equal variances.

\section{RESULTS AND DISCUSSION}

\section{Spleens of agouti mice are melanotic}

In our previous works we suggested that the phenomenon of splenic melanosis in mice is a reflex of melanogenesis in the skin (Plonka et al., 2005, Michalczyk et al., 2009, Michalczyk-Wetula et al., 2013). In mice, the skin melanin production is almost exclusively limited to the anagen hair follicles (Chase, 1955; Slominski \& Paus 1993; Slominski et al., 1994). Consequently, if there exists such a correlation, it must be somehow coupled with the hair cycle and hair pigmentation. And indeed, we have found that the efficiency of splenic melanosis in C57BL/6 mice is depended on normal, depilationinduced, and dystrophic hair cycle (Michalczyk-Wetula et al., 2013), and limited to the time period when the type of hair cycling is wave-like (i.e. ca. the first 6 months of life; Michalczyk et al., 2009). The differences in biophysical properties of melanin may suggest that the splenic pigment is heterogeneous and in general - more degraded that the one in the hair shafts (Plonka et al.,

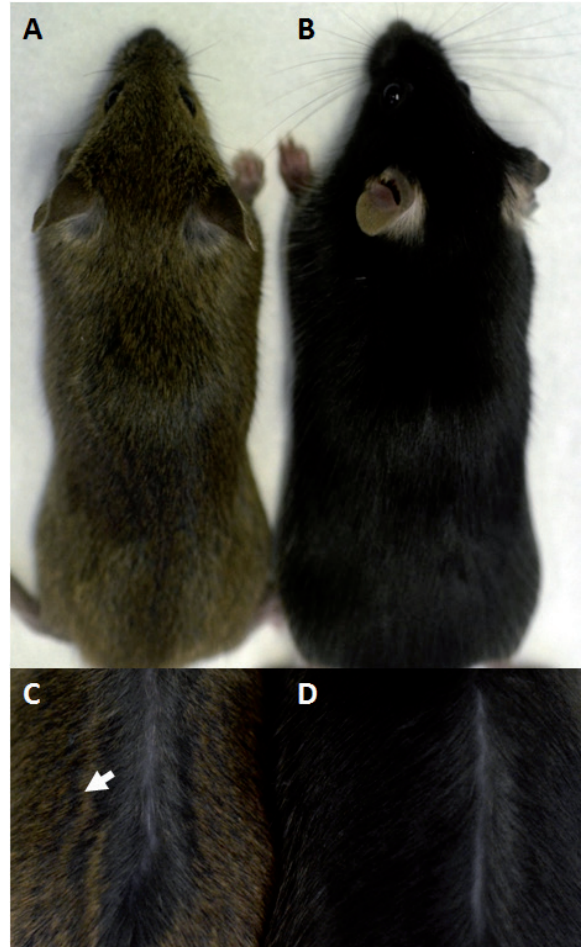

Figure 2. C57BL/6;129/SvJ mixed background mice with agouti (A) and black (B) fur color phenotype. The yellow "band" (arrow) close to the black tip of the hair shafts becomes visible after parting the agouti (C), but not black (D) back hair.

2005). Perhaps, spleen is the organ of ultimate melanin deposition and/or utilization or recycling.

Here we have found that the same phenomenon exists in the mice revealing the agouti phenotype. The mice in Fig. 2 possess the typical agouti fur coat (Fig. 2A), which is apparently brighter from the black phenotype (Fig. 2B), due to a break in the intensive eumelanogenesis which in black mice maintains its intensity during the whole anagen 6 (Plonka et al., 1995). This break is not a period totally devoid of melanogenesis, as then pheomelanogenesis dominates over eumelanogenesis, which results in a yellow "band" close to the black tip of the hair shaft (compare Fig. 2C and D; Sakurai et al., 1975; Oyehaug et al., 2002; Slominski et al., 2005a).

The pictures in Fig. 3 represent the spleens derived from agouti (Fig. 3A, B) and black (Fig. 3C) mice. The respective histological sections (FM and $\mathrm{HE}$ staining) are shown in Fig. 3D-K. This panel shows that the phenomenon in both phenotypes possesses a "yes-or-no" character, which has been many times reported before for black mice (Weissman, 1967; Plonka et al., 2005; Michalczyk et al., 2009; Michalczyk-Wetula et al., 2013). It also reveals that from the histological point of view, the phenomenon manifests itself similarly in the both mice phenotypes.

\section{EPR characteristics of melanotic spleens of agouti and black mice}

Figure 4 represents the X-band EPR spectra of the analyzed materials. All of them revealed a singlet signal around $\mathrm{g}=2.004$ which indicates a free radical-like paramagnetism typical of melanins (Commoner et al., 1954; Meredith \& Sarna, 2006; Plonka, 2009). Pheomelanins are known for revealing the ${ }^{14} \mathrm{~N}$ hyperfine splitting, as a consequence of semiquinonimine-like paramagnetic cen- 


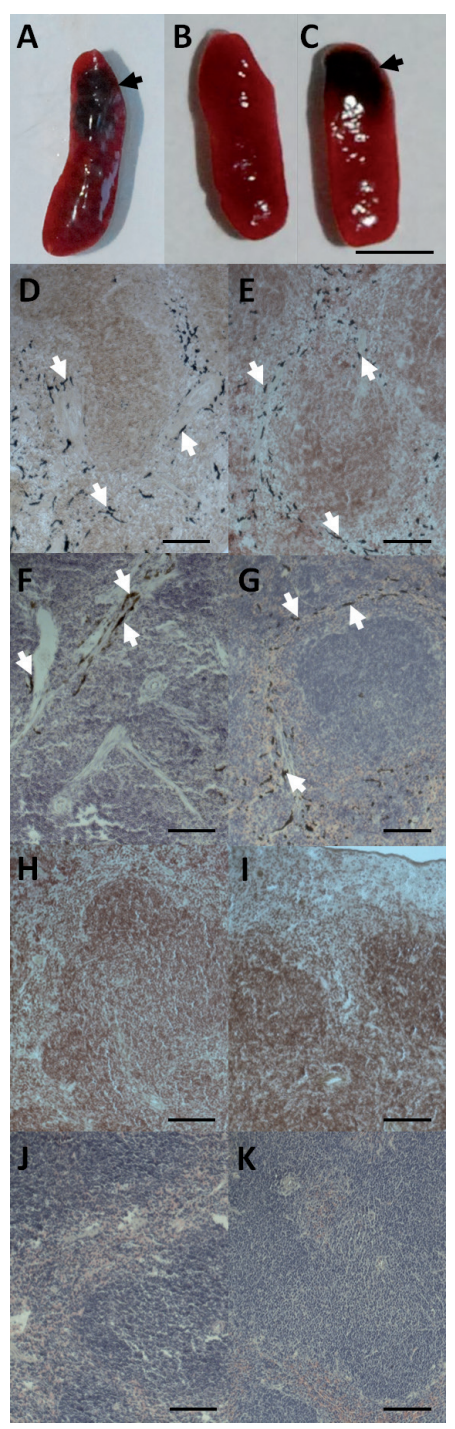

Figure 3. Macroscopic demonstration of representative melanosis (arrows) in spleens of agouti (A) and black (C) C57BL/6;129/ SvJ mixed background mice.

Some spleens in both groups were not pigmented (B). Histological manifestation of splenic melanosis in agouti (D, F), and black (E, G) C57BL/6;129/SvJ mixed background mice, and lack of melanin in spleens without melanosis in agouti $(\mathbf{H}, \mathbf{J})$, and black $(\mathbf{I}, \mathbf{K})$ mice. F, G, J, K - HE staining, D, E, H, I - FM staining. White arrows - melanin $(\mathbf{D}-\mathbf{G})$ in the melanotic $(\mathbf{A}, \mathbf{C})$ regions. Scale bars $-0.5 \mathrm{~cm}(\mathbf{A}-\mathbf{C}), 100 \mu \mathrm{m}(\mathbf{D}-\mathbf{K})$.

tres present in this polymer of benzothiazine-derivatives (Fig. 4C). Eumelanins do not contain nitrogen in the vicinity of unpaired electrons (the semiquinone-like paramagnetic centres), which results in the lack of the hyperfine splitting of the EPR signal (Fig. 4D) (Sealy et al., 1982). There are also other differences in the spectroscopic properties of the pigments (pheomelanin reveals a slightly wider signal and the g-value is slightly moved towards higher values; Sealy et al., 1982; Slominski et al., 2005a; Chikvaidze et al., 2014) but the hyperfine splitting is the most direct and easy to note feature of the EPR signal, characteristic for pheomelanins (see Fig. 4C, E, $\mathrm{G}, \mathrm{H})$. It must also be emphasized that the natural pigments usually are co-polymers of eu- and pheomelanin, and they often contain only admixture of pheomelaninlike centres (Ito \& Fujita, 1985). However, such admixture, if big enough, can be detected with EPR as a peak on the low-field side of the singlet (Vsevolodov et al.,

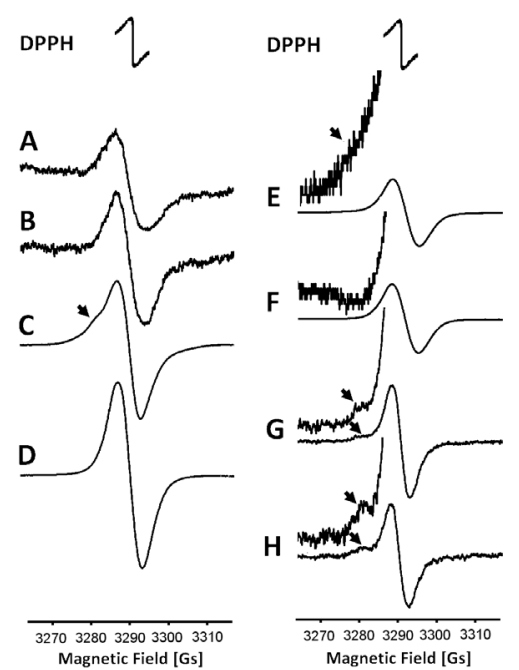

Figure 4. Representative EPR spectra of melanotic spleens, fur and synthetic pigments.

(A, B) melanized spleens of agouti (A) and black mice (B), cysteinyldopa (C), and dopa melanin (D) powder samples, agouti $(\mathbf{E})$, and black (F) mice fur, agouti hamster fur (G), and agouti gerbil fur (F). DPPH - the free radical standard signal. The pheomelanin component of the signals (arrow) was detectable only under high receiver gain (E-H - insets). The spectra were analyzed at $77 \mathrm{~K}$, at $\mathrm{X}$-band $(9.28 \mathrm{GHz})$, and variable receiver gain: $2 \times 10^{6}(\mathbf{A}, \mathbf{B}), 5 \times 10^{4}(\mathbf{C}), 1 \times 10^{4}(\mathbf{D}-\mathbf{F}), 4 \times 10^{5}(\mathbf{G}, \mathbf{H})$; insets: $1.5 \times 10^{5}(\mathbf{E})$ and $1.5 \times 10^{6}(\mathbf{F}-\mathbf{H})$; other parameters - see "Material and Methods".

1992; Hill et al., 1997; Plonka et al., 2003; Slominski et al., 2005b; Wolnicka-Glubisz et al, 2012).

The best method to detect and measure the content of eu- and pheomelanin-like constituents of a biological samples is based on the degradation of the material and detection of AHP (aminohydroxyphenylalanine) and PTCA (pyrrole-2,3,5-tricarboxylic acid) by HPLC (Ito \& Fujita, 1985). This method, however, reveals its limitations, though it has been shown to well correlate with EPR (Vsevolodov et al., 1991). EPR is easy to use when a big number of samples is studied, and it has turned out particularly suitable to measure melanins in hair (Ozeki et al., 1995; Plonka 2009; Chikvaidze et al., 2014). In the case of pre-domination of eumelanin, the strong singlet may mask the weak hyperfine features of the pheomelanin signal (Vsevolodov et al., 1991, Plon$\mathrm{ka} 2009$ ). But neither of the method is able to answer whether the material contains a co-polymer, or is just a mosaic of regions containing different proportions of eu-/and pheomelanin.

\section{Material derived of agouti mice contains low contribution of pheomelanin}

In our materials a clear contribution of pheomelanin could be detected only in the agouti fur of golden hamsters and Mongolian gerbils (Fig. 4G, H). In the back fur of the agouti mice only a trace of pheomelanin could be found in some samples (cf. Fig. 4E — inset), which reveals that the contribution of pheomelanin must be very low, however, higher than in the black murine fur (Fig. 4F). The same tendency is visible in the line widths $(4.79 \pm 0.07 \mathrm{Gs}$ for black and $4.80 \pm 0.08 \mathrm{Gs}$ for agouti mice). This stays in agreement with the data obtained from the same material analyzed by HPLC preceded by chemical degradation (Ozeki et al., 1995; 1996; Ito \& Wakamatsu, 2003). 
The quality of the splenic melanin does not allow for an unequivocal statement that it does contain pheomelanin (Fig. 4A). The intensity of the splenic signals is in general low even in the black animals (Fig. 4B). Because of other free radical signals resulting from the mitochondrial oxygen metabolism of the tissue (Davies \& Hochstein, 1982; Elas et al., 2008), or originating from blood infiltrating the spleen (Kubiak et al., 2013) and overlapping the melanin signal, the exact EPR measurement of splenic melanosis contains a considerable error. For this reason we expressed the splenic EPR signal intensity as the peak-to-peak amplitude, and we resigned from comparing the linewidths. While in some samples of spleens a trace of a low-field peak could be detected, the amelanotic spleens also revealed a wide and weak EPR signal (Michalczyk-Wetula et al., 2013; here - not shown), therefore the only conclusion that can be driven from the EPR results is that the melanotic spleens from the agouti mice tend to reveal a weaker EPR signal than the ones of black mice. This, however, quantitatively correlates well with the results of the EPR measurements of fur.

\section{Spleens of agouti mice tend to be weaker pigmented than of black mice}

Figure 5 contains the pooled results of the long-term observations of splenic melanosis in agouti and black animals. We have measured the percentage of macroscopically pigmented spleens in various experimental groups (these were particular groups of animals of similar age which were selected not to be bred any more). We were also able to estimate the pigmented area of each melanotic spleen (see Plonka et al., 2005; Michalczyk et al., 2009; Michalczyk-Wetula et al., 2013).

Both the percentage of the melanotic spleens and the average area of pigmented spots tended to be lower in the agouti mice (Fig. 5A, B - the lines of trend. Note that in the beginning, i.e. for young mice, the trend for agouti mice starts at the half of the initial value for the black mice - Fig. 5A). This correlates with the pooled quantitative data from spleens and fur (Fig. 6A-C), including the results of EPR measurements of spleens (Fig. 6B). Taking into consideration the age of animals, we have checked the splenic index and the absolute weights of the mice and the spleens. The splenic melanosis did not affect the spleen weight, nor the splenic index in any of the mice phenotypes (not shown). We also did not observe any difference between the average mass or splenic index between the age-matched mice of various fur phenotypes (not shown). Therefore, we decided not to standardize the EPR signal amplitudes per a stable mass of spleens, and our quantitative results represent the total amount of melanin in a spleen.

We also calculated the total percentage of pigmented spleens in agouti $(12 \%)$ and in black mice (19.7\%). Such comparison is only in part useful, because starting from ca. $20^{\text {th }}-25^{\text {th }}$ week of life, the spleens become amelanotic (Fig. 5A,B); but the tendency is preserved anyway.

We expected a stronger difference in pigmentation of the materials delivered from the agouti and black mice, but the objective, physical method - EPR, despite apparently brighter superficial coloration of the fur coat (Fig. 2), unexpectedly revealed that the agouti fur of mice contains only insignificantly lower amount of melanin than the black fur. The same observation was made with HPLC/chemical degradation method (Ozeki et al., 1995; 1996; Ito \& Wakamatsu 2003). The
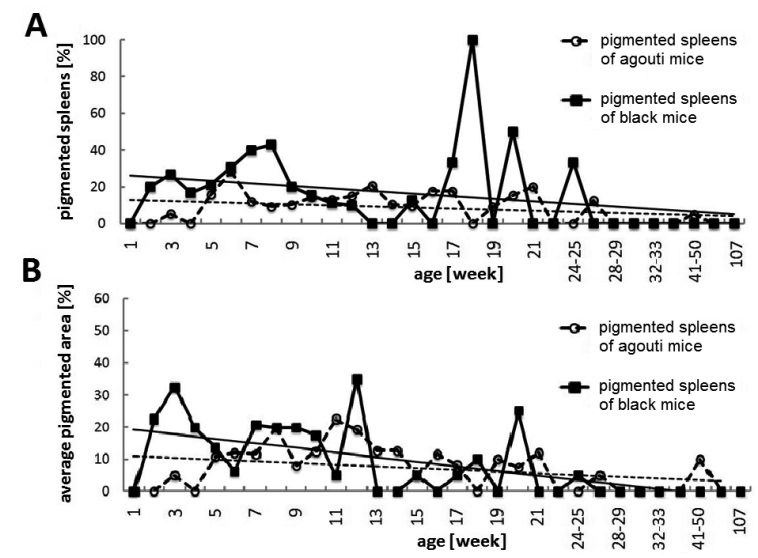

C

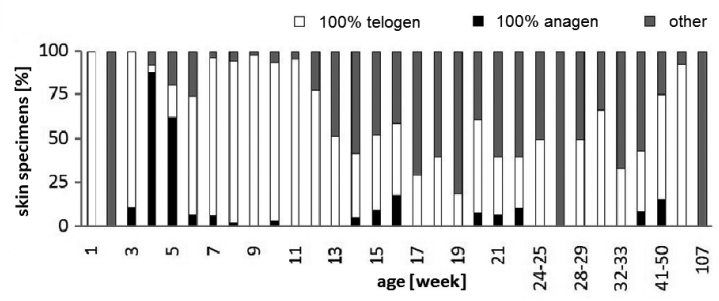

D

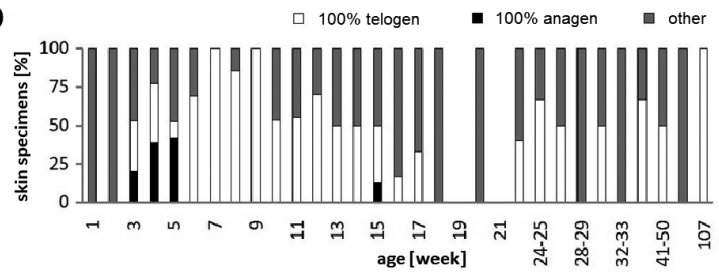

Figure 5. Pigmentation of spleens on subsequent weeks of life, and the corresponding stage of hair cycle in the back skin in agouti and black mice.

(A) percentage of melanotic spleens in a given age group. (B) corresponding average area of melanosis in the melanotic spleens. (C, D) percentage of mice with the whole back skin in anagen VI, whole back skin in telogen, or with the back skin in various stages of the hair cycle (anagen-catagen or telogen-anagen transition or black spots in telogen skin), in agouti (C) or black mice (D). Open circles and dashed line - agouti, solid squares and solid line black mice. The linear trend lines are also shown.

actual bright band is maintained only near to the surface of the fur coat, while the deeper parts of the hair remain black. This can be seen when parting delicately the hair until the skin is visible (see Fig. 2C). If this tendency is preserved also for spleens, only long-time observations would make us possible to catch the weak difference in pigmentation between agouti- and black-mice-derived spleens (Fig. 5A, B - the lines of trends).

We were not able to find any statistical significance between the average pigmentation of agouti and black mice, their spleens nor fur. However, the data obtained by several independent methods and observables, based on different physical rationales, and the trends, reveal the same tendency. Therefore, the results entitle us to draw the conclusion that the splenic melanosis in agouti mice is slightly weaker, as compared to the black counterparts. This is another argument for the thesis that the splenic melanosis correlates well with the fur pigmentation in mice. 

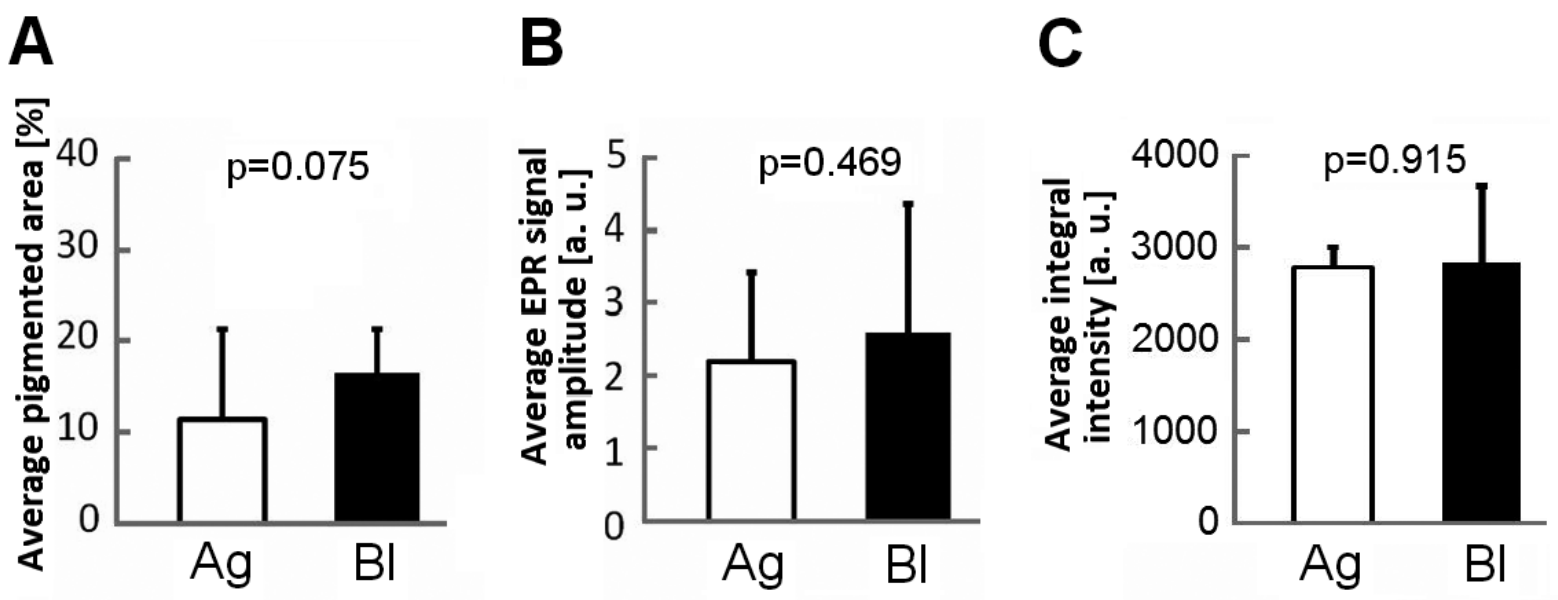

Figure 6. Pooled results concerning pigmentation.

(A) average area of splenic melanosis, (B) average EPR signal amplitude of spleens, (C) average integral EPR signal intensity of fur. Empty bars - 129/SvJ agouti mice, solid bars - C57BL/6 black mice. The means of $n=38$ (black mice ) or $n=93$ (agouti mice) melanotic spleens (A), $n=15$ spleens (each phenotype, spleen EPR signal - B), $n=4$ (agouti mice) and $n=8$ (black mice) fur samples (A) \pm S.D. (B) receiver gain $-1 \times 10^{6},(\mathbf{C})$ receiver gain 1.25 $\times 10^{4}$; other details concerning the EPR measurements - see "Material and Methods".

\section{Correlation of splenic melanosis with hair cycle is difficult to detect for spontaneous hair cycling}

We tried to correlate the splenic melanosis with the stages of the hair cycle, which we roughly determined on the base of the bottom surface of the back skin removed from the animals (Fig. 1). This is the first paper in which we report such mode of determining the area and the progress of hair cycle in mice. It is very quick, and allows one to use the skin for other purposes, but of course, it does not replace the histomorphometry, which delivers detailed information on the percentage of hair follicles in a given stage of hair cycle (Michalczyk et al., 2009). Nevertheless, we could not find any sharp, and systematic differences between melanosis of the mice sacrificed on subsequent weeks after birth (Fig. 5C, D), as we did previously (Plonka et al., 2005; Michalczyk-Wetula, 2013). The shape of the curves in Fig 5A, B were only roughly correlated with each other, and only in a very general way reflected the previous findings. This can be, however explained by the fact that here the spontaneous cycle was investigated in randomly assembled groups of mice (which did not came from the same nests, as in the paper by Plonka et al., 2005). In young mice the mode of hair cycling is wave-like, but the onset of the subsequent anagen stages is quite random within some limits (Dry 1926; Chase \& Eaton, 1959; Militzer 2001; Plonka et al., 2008).

\section{CONCLUSIONS}

Splenic melanosis does appear in the agouti mice, but, like the fur pigmentation, it is only slightly weaker than in the black mice. It seems, consequently, to accurately reflect the hair coat melanization in the both phenotypes of mice. The agouti phenotype is the basic, cryptic phenotype of small wild mammals, like rodents, which serves for them as a perfect camouflage (Wood et al., 1999). While there are numerous exceptions of this tendency, it seems convincing that the hair pigmentationrelated splenic melanosis is not an exotic peculiarity observed only in some inbred strains of the animals bred in laboratories, but a phenomenon which must take place in the wild nature, perhaps on a pan-mammalian scale.

\section{Acknowledgements}

The authors are grateful to Professors Joanna Cichy and Krystyna Urbańska for their kind support, and to Norbert Wolan for his help at performing EPR measurements. Faculty of Biochemistry, Biophysics and Biotechnology of Jagiellonian University is a partner of the Leading National Research Center (KNOW) supported by the Ministry of Science and Higher Education.

\section{Conflict of Interest}

The authors declare no conflict of interest.

\section{REFERENCES}

Bultman S, Michaud E, Woychik R (1992) Molecular characterization of the mouse agouti locus. Cell 71: 1195-1204.

Chikvaidze EN, Partskhaladze TM, Gogoladze TV (2014) Electron spin resonance (ESR/EPR) of free radicals observed in human red hair: a new, simple empirical method of determination of pheomelanin/eumelanin ratio in hair. Magn Reson Chem 52: 377-382.

Commoner B, Townsend J, Pake GW (1954) Free radicals in biological materials. Nature 174: 689-691.

Chase HB (1954) Growth of the hair. Physiol Rev 34: 113-126.

Chase HB, Eaton GJ (1959) The growth of hair follicles in waves. Ann N Y Acad Sci 83: 365-368.

Crichton DN, Busuttil A, Price WH (1978) Splenic lipofuscinosis in mice. J Pathol 126: 113-120.

Davies KJA, Hochstein P (1982) Ubisemiquinone radicals in liver: implications from a mitochondrial Q cycle in vivo. Biochem Biophys Res Commun 107: 1292-1299.

Dry FW (1926) The coat of the mouse (Mus musculus). J Genet 16: 281340 .

Elas M, Bielanska J, Pustelny K, Plonka PM, Drelicharz L, Skorka T, Tyrankiewicz U, Wozniak M, Heinze-Paluchowska S, Walski M, Wojnar L, Fortin D, Ventura-Clapier R, Chlopicki S (2008) Detection of mitochondrial dysfunction by EPR technique in mouse model of dilated cardiomyopathy. Free Radic Biol Med 45: 321-328.

Hill HZ, Hill GJ, Cieszka K, Plonka PM, Mitchell DL, Meyehoffer MF, Xin P, Boissy RE (1997) Comparative action spectrum for ultraviolet light killing of mouse melanocytes from different genetic coat color backgrounds. Photochem Photobiol 65: 983-989.

Ito S, Fujita K (1985) Microanalysis of eumelanin and pheomelanin in hair and melanomas by chemical degradation and liquid chromatography. Anal Biochem 144: 527-536. 
Ito S, Wakamatsu K (2003) Quantitative analysis of eumelanin and pheomelanin in humans, mice, and other animals: a comparative review. Pigment Cell Res 16: 523-531.

Kaelin CB, Candille SI, Yu B, Jackson P, Thompson DA, Nix MA, Binkley J, Millhauser GL, Barsh GS (2008) New ligands for melanocortin receptors. Int J Obesity 32: S19-S27.

Kubiak T, Krzyminiewski R, Dobosz B (2013) EPR study of paramagnetic centers in human blood. Curr Top Biophys 36: 7-13.

Land EJ, Ramsden CA, Riley PA (2004) Quinone chemistry and melanogenesis. Methods Ensymol 378: 88-109.

Linden M, Ward JM, Cherian S (2012) Hematopoietic and lymphoid tissues. In Comparative anatomy and bistology. A mouse and human atlas. Treuting MP, Dintzis SM, eds. pp 309-338. Academic Press@ Elsevier, Amsterdam, Boston, Heidelberg, London, New York, Oxford, Paris, San Diego, San Francisco, Singapore, Sydney, Tokyo.

Linder CC (2003) Mouse nomenclature and maintenance of genetically engineered mice. Comp Med 53: 119-125.

Meredith P, Sarna T (2006) The physical and chemical properties of eumelanin. Pigment Cell Res 19: 572-594.

Michalczyk D, Popik M, Salwinski A, Plonka PM (2009) Extradermal melanin transfer? Lack of macroscopic spleen melanization in old C57BL/6 mice with de-synchronized hair cycle. Acta Biochim Pol 56: 343-353.

Michalczyk-Wetula D, Salwiński A, Popik M, Jakubowska M, Plonka PM (2013) Splenic melanosis during normal murine C57BL/6 hair cycle and after chemotherapy. Acta Biochim Pol 60: 313-321.

Militzer K (2001) Hair growth pattern in nude mice. Cells Tissues Organs 168: $285-294$.

Millar SE, Miller MW, Stevens ME, Barsh GS (1995) Expression and transgenic studies of the mouse agouti gene provide insight into the mechanisms by which mammalian coat color patterns are generated. Development 121: 3223-3232.

Ollmann MM, Lamoreux LL, Wilson BD, Barsh GS (1998) Interaction of Agouti protein with the melanocortin 1 receptor in vitro and in vivo. Gene Dev 12: 316-330.

Oyehaug L, Plahte E, Vage, DI, Omholt SW (2002) The regulatory basis of melanogenic switching. J Theor Biol 215: 449-468.

Ozeki H, Ito S, Wakamatsu K, Hirobe T (1995) Chemical characterization of hair melanins in various coat-color mutants of mice. J Invest Dermatol 105: 361-366.

Ozeki H, Ito S, Wakamatsu K, Thody AJ (1996) Spectrophotometric characterization of eumelanin and pheomelanin in hair. Pigment Cell Res 9: 265-270.

Paus R, Christoph T, Müller-Röver S (1999) Immunology of the hair follicle: a short journey into terra incognita. I Investig Dermatol Symp Proc 4: 226-234.

Plonka PM (2009) Electron paramagnetic resonance as a unique tool for skin and hair research. Methods review. Exp Dermatol 18: 472484.

Plonka PM, Michalczyk D, Popik M, Handjiski B, Slominski A, Paus R (2005) Splenic eumelanin differs from hair eumelanin in C57BL/6 mice. Acta Biochim Pol 52: 433-441.

Plonka PM, Michalczyk D, Popik M, Handjiski B, Paus R (2008) Electron paramagnetic resonance (EPR) spectroscopy for investigating murine telogen skin after a spontaneous or depilation-induced hair growth. J Dermatol Sci 49: 227-240.

Plonka PM, Passeron T, Brenner M, Tobin D, Shibahara S, Thomas A, Slominski A, Kadekaro AL, Hershkovitz D, Peters E, Nordlund JJ, Abdel-Malek Z, Takeda K, Paus R, Ortonne JP, Hearing VJ, Schallreuter $\mathrm{K}$ (2009) What are melanocytes really doing all day long...? Exp Dermatol 18: 799-819.
Plonka P, Plonka B, Paus R (1995) Biophysical monitoring of melanogenesis as a tool for pigment and hair research. Arch Dermatol Res 287: 687-690.

Plonka PM, Slominski AT, Pajak S, Urbanska K (2003) Transplantable melanomas in gerbils (Meriones unguiculatus). II - Melanogenesis. Exp Dermatol 12: 356-364.

Sakurai T, Ochiai H, Takeuchi T (1975). Ultrastructural change of melanosomes associated with agouti pattern formation in mouse hair. Dev Biol 47: 466-471.

Schallreuter KU, Kothari S, Chavan B, Spencer JD (2008) Regulation of melanogenesis - controversies and new concepts. Exp Dermatol 17: 395-404.

Sealy RC, Hyde JS, Felix CC, Menon IA, Prota G, Swartz HM, Persad $S$, Haberman HF (1982) Novel free radicals in synthetic and natural pheomelanins: distinction between dopa melanins and cysteinyldopa melanins by ESR spectroscopy. Proc Natl Acad Sci USA 79: 2885-2889.

Slominski A, Goodman-Snitkoff G, Maurer M, Paus R (1997) Hair cycle-associated changes in splenocyte proliferation. In Vivo 11: 101-102.

Slominski A, Paus R (1993) Melanogenesis is coupled to murine anagen: towards new concepts for the role of melanocytes and the regulation of melanogenesis in hair growth. I Invest Dermatol 101: 90S-97S.

Slominski A, Paus R, Plonka P, Chakraborty A, Maurer M, Pruski D, Lukiewicz S (1994) Melanogenesis during the anagen-catagen-telogen transformation of the murine hair cycle. I Invest Dermatol 102: 862-869.

Slominski A, Plonka PM, Pisarchik A, Smart JL, Tolle V, Wortsman J, Low MJ (2005a) Preservation of eumelanin hair pigmentation in proopiomelanocortin-deficient mice on a nonagouti (a/a) genetic background. Endocrinology 146: 1245-1253.

Slominski A, Tobin D J, Shibahara S, Wortsman J (2004) Melanin pigmentation in mammalian skin and its hormonal regulation. Physiol Rev 84: 1155-1228.

Slominski A, Wortsman J, Plonka PM, Schallreuter KU, Paus R, Tobin DJ (2005b) Hair follicle pigmentation. J Invest Dermatol 124: 13-21.

Threadgill DW, Yee D, Matin A, Nadeau JH, Magnuson T (1997) Genealogy of the 129 inbred strains: $129 / \mathrm{SvJ}$ is a contaminated inbred strain. Mamm Genome 8: 390-393.

Vachtenheim J, Borovanský J (2010) "Transcription physiology" of pigment formation in melanocytes: central role of MITF. Exp Dermatol 19: $617-627$.

van der Heijden A, van Dijk JE, Lemmens AG, Beynen AC (1995) Spleen pigmentation in young C57BL mice caused by accumulation of melanin. Labor Anim 29: 459-463.

Vsevolodov EB, Ito S, Wakamatsu K, Kuchina II, Latypov IF (1991) Comparative analysis of hair melanins by chemical and electron spin resonance methods. Pigment Cell Res 3: 30-34.

Weissman I (1967) Genetic and histochemical studies on mouse spleen black spots. Nature 215: 315.

Wolnicka-Glubisz A, Pecio A, Podkowa D, Kolodziejczyk LM, Plonka PM (2011) Pheomelanin in the skin of Hymenochirus boettgeri (Amphibia: Anura: Pipidae). Exp Dermatol 21: 537-540.

Wood JM, Jimbow K, Boissy RE, Slominski A, Plonka PM, Slawinski J, Wortsman J, Tosk J (1999) What is the use of generating melanin? Exp Dermatol 8: 153-164.

Zieri R, Franco-Belussi L, de Souza Santos LR, Taboga SR, de Oliveira C (2015) Sex hormones change visceral pigmentation in Eupemphix nattereri (Anura): effects in testicular melanocytes and hepatic melanomacrophages. Anim Biol 65: 21-32. 